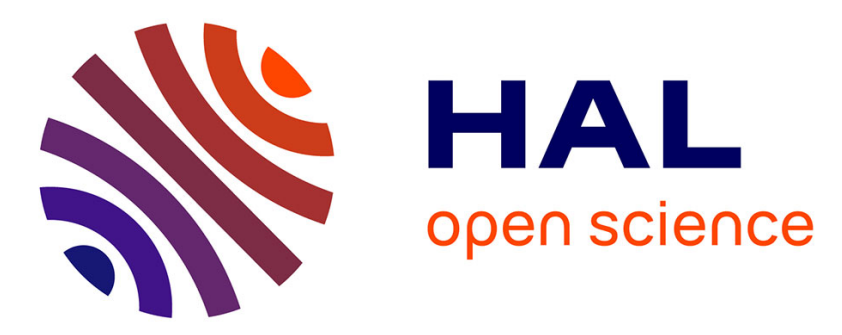

\title{
Absorptive Resilience Phase Assessment Based on Criticality Performance indicators for Water Distribution Networks
}

David Ayala Cabrera, Olivier Piller, Manuel Herrera, Denis Gilbert, Jochen Deuerlein

\section{To cite this version:}

David Ayala Cabrera, Olivier Piller, Manuel Herrera, Denis Gilbert, Jochen Deuerlein. Absorptive Resilience Phase Assessment Based on Criticality Performance indicators for Water Distribution Networks. Journal of Water Resources Planning and Management, 2019, 149 (9), 04019037, 15 p. 10.1061/(ASCE)WR.1943-5452.0001097 . hal-02264092

\section{HAL Id: hal-02264092 \\ https://hal.science/hal-02264092}

Submitted on 6 Aug 2019

HAL is a multi-disciplinary open access archive for the deposit and dissemination of scientific research documents, whether they are published or not. The documents may come from teaching and research institutions in France or abroad, or from public or private research centers.
L'archive ouverte pluridisciplinaire $\mathbf{H A L}$, est destinée au dépôt et à la diffusion de documents scientifiques de niveau recherche, publiés ou non, émanant des établissements d'enseignement et de recherche français ou étrangers, des laboratoires publics ou privés. 

2019, 149 (9), 15 p.

The original publication is available at https:// ascelibrary.org/ doi/full/10.1061/(ASCE)WR.1943-5452.0001097s Doi : 10.1061/(ASCE)WR.1943-5452.0001097

\title{
Absorptive Resilience Phase Assessment Based on Criticality Performance Indicators for Water Distribution Networks
}

\section{David Ayala-Cabrera $^{1}$, Olivier Piller ${ }^{2}$, Manuel Herrera ${ }^{3}$, Denis Gilbert ${ }^{4}$, and Jochen Deuerlein ${ }^{5}$}

${ }^{1}$ Postdoctoral Research Fellow, Dept. of Water, Irstea, F-33612 Cestas, France. Doctor of Engineering, Ingeniousware GmbH, Jollystraße 11, 76137 Karlsruhe, Germany.

Email: david.ayala@irstea.fr

${ }^{2}$ Senior Research Scientist, Irstea, Dept. of Water, Bordeaux Regional Centre, UR ETBX, 50 Ave. de Verdun, Gazinet, F-33612 Cestas, France. Adjunct Senior Lecturer, School of Civil, Environmental, and Mining Eng., Univ. of Adelaide, Adelaide, SA 5005, Australia.

${ }^{3}$ Research Associate, Dept. of Engineering, University of Cambridge, 17 Charles Babbage Rd., CB3 0FS Cambridge, United Kingdom.

${ }^{4}$ Research Engineer, Irstea, Dept. of Water, Bordeaux Regional Centre, UR ETBX, 50 Ave. de Verdun, Gazinet, F-33612 Cestas, France.

${ }^{5}$ Senior Researcher, 3S Consult GmbH, D 76137 Karlsruhe, Germany. Adjunct Senior Lecturer, School of Civil, Environmental, and Mining Eng., Univ. of Adelaide, Adelaide, SA 5005, Australia.

\begin{abstract}
Water distributions networks (WDNs) are exposed to multiple hazards leading the network to operate under a range of critical conditions. This paper explores the relationship between the impact of anomalous events (AEs) of WDNs and the consequent palliative actions (PAs) to be implemented in the network to minimize such impact. Both AEs and PAs are assessed through a network resilience criticality index adapted to WDNs. The results are compared to those obtained from normal operating conditions with respect to the satisfaction rate of nodal demands. The
\end{abstract}


proposal is evaluated by two case-studies. The first corresponds to a small synthetic network and the second to a medium size utility network. After a pipe burst event analysis there are also scrutinized two different isolation actions in each of the two WDNs. The results quantify system's resilience and support water utility managers on further decision-making processes. This is done through critical resilience indicators that show to provide information and support both for better crisis preparedness (planning) and management (mitigation).

\section{INTRODUCTION}

Water distribution networks (WDNs) are key urban complex infrastructures. They provide an essential resource for life, being considered as critical infrastructures that require protection to adequately accomplish their service (?). In 2014, a pipe burst of one of Los Angeles' (California, USA) main pipes left water losses over 5,600 1/s. In 2018 and by similar reason, roads were closed to motorists and pedestrian in central London (UK) while more than 20,000 homes were affected by water shortage. Also in 2018, big factories such as Jaguar, Land Rover, and Cadbury shut plants so water firm can fix burst pipes originated by cold weather in the West Midlands (UK). These examples show how ensuring WDN resilience and security is a big concern for water utilities.

Water network vulnerability to failures depends on several factors such as nature of the affected consumers, assets location, and time of the event occurrence. A continuous water supply service has better security than those having intermittent service (?). In this regard, several authors argue in their research that the best manner to guarantee water quality is by maintaining a pressure head above a target threshold and preserving the continuity of the supply through the network (?; ?; ?). Assessing WDN node importance plays a key role for approaching vulnerability. It depends on a number of factors: sensitive population, node location, and system performance. Regarding pipes, first we should notice is that they might perform a very different role for the water supply ranging from distribute water to other pipes to properly supply consumers (?). Thereby, it is clear that some of the network pipes are more important than others from a hydraulic point of view. Thus, recognizing the diverse and relative importance of its pipes it is an essential factor to evaluate the overall hydraulic performance (?). Pipes importance is related to estimate the risk of network 
isolation and the risk of deficient pressure head at the demand points. ?) argue that information related to the pipe importance will be helpful for design, planning, control and WDN management. Several studies (?; ?) focus their attention on the evaluation of the network performance under pipe failures. Authors prioritized in this way network links by studying how each link failure would affect the post-anomalous event network performance $(? ; ? ; ;)$. Multiple failures of the components is another issue with high relevance in resilience studies. Widely considered causes of multiple failures in pipes are the location of the isolating valves (isolation of surrounding area of the affected pipe) (?), natural disasters (?), severe failures due to human-made causes (?), sudden-onset disaster that are followed by multiple related sub-disasters (cascading events) (?), and random multiple failures (?). It is, then, necessary to ensure a satisfactory performance of the system by planing and investment on the study of those components critical for a suitable water supply (?).

In a WDN context, the first definition of resilience was given by (?). In their work, some criteria were proposed for describing the performance in terms of reliability, resilience and vulnerability. Resilience refers to the strength of the network and its behavior under different anomalous events (AEs) (?; ?). The ability of the system to resist stress scenarios, mitigate failures, and overcome their consequences through a quick recovery is often referred to as the resilience of the system (?). Thus, network managers require implement actions to support their decision-making process towards more resilient systems (?). WDN resilience assessment focuses on either the mechanical failure of components such as pipe or pump failure. It should also be considered any hydraulic failure of the system due to degraded pipe capacities and/or to uncertain nodal demand flows (?). Resilience criticality indicators (RCIs) seek to quantify the impact on consumers as consequence of an anomaly that occurs within the WDN. The criticality of the pipes (and other WDN assets) is measured through the impact on the disruption to supply (?). In essence, RCIs attempt to evaluate the capability of the system under different abnormal operational conditions such as emergencies, component failures, and hydraulic changes. RCIs quantify how resilient the system is and support the decision on actions in order to reduce the occurrences and to minimize the possible consequences of any AE. 
This work proposes a tool for exploring the consequences of pipe failures with respect to the hydraulic performance of a WDN. This methodology can be adapted to the kind of critical event that occurs (mechanical or hydraulic failure) and to the type of operative actions taken on response at such event (mitigation, adaptation, restoration). The method splits into five stages: a) Define network model and consumer characteristics, b) run hydraulic computer models, c) assess system performance state, d) quantify resilience through RCIs, and e) resilience visualization. The proposal aims to be a reliable tool to quantify the system resilience, supporting the decision-making process to eventually reduce the occurrence of failures and minimize their potential consequences.

The paper also characterizes how the network works under pipe burst scenarios. Several positions for pipe burst occurrences are evaluated. This is approached by considering water demand at peak and valley requirements. Afterwards, the impact on the network performance is assessed through an RCI specifically tailored to WDNs and also compared to normal operating conditions regarding the satisfaction rate of nodes. The system resilience is presented as maps of component importance. These maps represent the average system impact as consequence of $\mathrm{AE}$ in each pipe of the WDN. These maps provide an easy and quick identification of areas of high importance components in the WDN. This is made through visualization of both the overall effects of any disruption event and the extent of low resilience CIs areas. The resilience assessment is complemented by the so-called palliative actions (PAs) in case of disruption events. PAs operates single and multiple isolation of WDN areas. The isolation PAs are mitigation actions attempting to minimize the potential negative effects related to an AE. It is possible, then, to determine how to enhance the network performance facing an $\mathrm{AE}$ by to the implementation of these types of isolation actions and comparing the most critical results after application of each PA (for a specific time).

\section{PROPOSED THEORETICAL FRAMEWORK}

There is a need to develop a generic framework associated to WDN resilience as this concept remains unclear in literature. This is based on the three-stage resilience approaches proposed by the Franco-German ResiWater Project (?). These three-stages are supported by the three-capacities of the system (?): 1) absorptive capacity, 2) adaptive capacity, and 3) restorative capacity. The 
first capacity refers to the ability of the system to absorb the impact of any system perturbation and to minimize its consequences (without corrective action from water utility managers side). The adaptive capacity is the ability of the system to temporarily adjust undesirable situations by undergoing some changes if absorptive capacity has been exceeded. The restorative phase refers to the capacity of the system to implement long-term solutions so that the system performance reaches a stable or better level than the initial state (prior to adverse operative conditions) (?).

The resilience notion aims at developing tools to prepare water utilities for crisis scenarios; as it is the case of ResiWater project (?). This improves the definition proposed by ?) by including the criterion of preparedness. Resilience, and specifically WDN resilience, is often measured using performance metrics. Fig. ??a shows an example of a performance-based resilience curve, also called "functionality curve" or "resilience triangle" (see e.g. ?; ?). The horizontal axis represents time and the vertical axis performance (criteria to assessing resilience) (?). This resilience curve can be split into events and actions. The first division of the resilience curve is measured at the event starting time $\left(t_{\text {event }}\right)$ and goes until the water utility initiate appropriate (palliative) actions. Thus, we have the time in which the anomalous event occurs; $t_{\text {event }}$ (e.g. pipe burst). We also have the detection time $\left(t_{d e t}\right)$, and the starting time $t_{\text {pall }}$ in which any PA is implemented by the water utility. The times of the absorptive stage involved in the model (event(s) part of the resilience curve) are shown in Fig. $? ? \mathrm{~b}\left(t_{\text {event }} \rightarrow(+) t_{\text {det }} \rightarrow(+) t_{\text {pall }}\right)$. According to the framework of the ResiWater project, the first stage (absorptive phase) of the network's resilience is measured since $t_{\text {event }}$ occurs and goes until $t_{\text {pall }}$ (see Fig. ??b). It is also possible to quantify the internal vulnerability of the system mirroring its absorptive capacity. The absorptive phase is followed by another two stages (adaptive and restorative) (?). The times involved in the model (action(s) part of the resilience curve) are: $t_{s t a b}$, the time when all emergency measures are in place for maintaining the system performance; $t_{\text {end }}$, the time when the system performance reaches a stable level; $t_{a c c}$ or acceptable time, the maximum stipulated time in which the network can be under failure. The main goal for $t_{a c c}$ is to quantify the degree of severity that users suffer during the period in which the system is on failure mode. 
This paper focuses on characterizing a WDN working under a pipe burst scenario (without corrective action from water utility) at a specific time. According to the previous definition, the paper explores the most critical AE consequences at the absorptive phase of the network performance. This is made by shifting pipe breaks location along each WDN pipe and also varying the water demands. The paper also proposes to evaluate the isolation actions at the same demand period explored for the AE (see Fig. ??a) in order to determine the WDN capacity enhancing its performance and to explore the relative importance of pipes. To ranking pipes it is important to investigate the system performance during and after $t_{\text {pall }}$. However, this paper exclusively explores the functioning of the network at $t_{\text {pall }}$, due that this information allows to know the more real requirements for the WDN at the most critical operating condition during the absorptive phase. This also ease to compare the results with the starting of the adaptive phase. When the pressure head drops under unfavorable values, water utilities increase the pumping pressure (if pumps are available) in order to boost pressure across the system to maintain positive pressure heads and also avoid further contamination. These adaptive actions are only implemented prior to any palliative actions and aim to improve the ability of the system to keep working properly. In case of pressures back to normal, these actions delimit the end of the absorptive phase (at $t_{\text {pall }}$ ). Fig. ?? presents the flowchart for the proposed system for evaluating resilience. Each stage is explained in the following sections.

\section{Brief Description of the AE Under Investigation and Isolation Actions}

The AE under study corresponds to the pipe burst tested at each pipe of the system. There are evaluated three different burst along the pipe. These are: 1) at pipe's initial point (Pos0), 2) at pipe's center (Pos50), and 3) at pipe's terminal point (Pos100). The proposed burst locations proposed in this paper attempt to emulate those commonly used for hydraulic simulations in WDN (?; ?; ?; ?). Our proposal takes into account the flow direction within the pipes determining the burst locations (Pos0, Pos50, and Pos100). This corresponds to the flow orientation obtained through the simulation of the network working under normal operating conditions.

There are considered two isolation actions: 1) the single isolation action (SIA) of the affected 
pipe, and 2) the multiple isolation action (MIA) - isolation of the surrounding area of the affected pipe.

- SIA corresponds to the pipe isolation by means of two valves placed at the pipe extremes. This allows to close exclusively the affected pipe (?). ? proposed single pipe isolation as reliability indicator but it has a lack of practicality since any operation to fix a broken pipe involves the closure of a larger hydraulic segment. The $m-1$ reliability importance of each single pipe can be calculated by simulating the system without this link. The importance is defined as the ratio of the actual delivery and the target demand (see Section ??). This information aids to quantify the system resilience by means of RCIs and to make preventive actions, such as twinning of the most critical pipes, establishing schemes and prioritizing maintenance or rehabilitation actions, or possible removal of redundant pipes.

- MIA corresponds to the isolation of a surrounding to the affected broken pipe. MIA can occur in the network due to the isolation valves' configuration, where the exact location of the isolation valves is unknown (or it is necessary to close more than one pipe). MIA shows its usefulness as palliative action. The isolation of even larger parts of the network (combination of several hydraulic segments) may require MIA facing contamination events. This is depending on the source and its estimated spreading through the network. In this way, it is firstly stopped any further dissemination of the contaminant. This is followed by flushing of the contaminated pipes (?; ?; ?; ?). This action is applied to renew the water and extract the contaminant substance of the affected pipes and to additionally avoid the contaminant spread (?).

\section{Topological Characteristics of the Network}

WDNs can be represented as a network/graph $\mathbf{G}=\mathbf{G}(\mathbf{V}, \mathbf{E})$ of nodes/vertices (e.g. reservoirs, tanks, demand nodes), V, connected by links/edges (e.g. pipes, valves), E (?; ?). The topological characteristics of the networks are generally represented by the incidence matrix $\mathbf{A}^{\mathbf{N}}$, Eq. (??). 


$$
A_{(i, j)}^{N}=\left\{\begin{array}{cl}
-1 & \text { if node } i \text { is terminal point of link } j \\
0 & \text { if node } i \text { is not connected to link } j \\
1 & \text { if node } i \text { is the initial point of link } j
\end{array},\right.
$$

where $i=1, \ldots, n$ with $i$ the node number and $j=1, \ldots, m$, with $j$ the link number. For further hydraulic computations, matrix $\mathbf{A}^{\mathbf{N}}$ is generally partitioned into two sub-matrices, $\mathbf{A}_{\mathbf{f}}$ and $\mathbf{A}$; that represent respectively nodes with fixed head (reservoirs or tanks) and nodes with unknown head (demand or junction nodes). This incidence matrix maybe used to accelerate the detection of the surrounding area to the affected pipe (Appendix ??).

\section{Hydraulic Models}

The steady-state of the entire WDN is simulated both through the potential at the nodes (head) and the link flows at a specific time (?). This is expressed by the Eq. (??). In this equation, q represents the vector of flow in the links; $\mathbf{h}$ and $\mathbf{h}_{\mathbf{f}}$ are the head at nodes with unknown head, and head at fixed head nodes, respectively; and $\mathbf{d}$ represents the water demands at consumers (vector). The head losses in the links is described by $\Delta h(\mathbf{r}, \mathbf{q})$, where $\mathbf{r}$ is the pipe friction coefficient.

$$
\left\{\begin{array}{ll}
\mathbf{A q}+\mathbf{d}=0 ; & \text { mass balance at every node } \\
\Delta h(\mathbf{r}, \mathbf{q})-\mathbf{A}^{\mathbf{T}} \mathbf{h}-\mathbf{A}_{\mathbf{f}}{ }^{\mathbf{T}} \mathbf{h}_{\mathbf{f}}=0 ; & \text { energy balance at every link }
\end{array},\right.
$$

For hydraulic modeling of the network it is important to differentiate between the two demand driven model-DDM, and pressure driven model -PDM. Classical techniques for hydraulic analysis of WDNs (DDM formulations) are analyzed under the assumption that water demands are known and fully satisfied. This happens whether or not the available head pressure is enough to guarantee the delivered outflow. Hence, in the hydraulic simulations carry on through the uses of DDM approach, the delivered outflow (c) at each node $i$, is equal to the required design $\left(c_{i}=d_{i}\right)(\boldsymbol{?})$. If the pressure head drops below a certain threshold, as consequence of some $\mathrm{AE}$, the outflow rate will be significantly reduced. In those cases, the hydraulic analysis performed through DDM approaches can deviate considerably from reality (?; ?). 
For a PDM approach (suitable for systems operating under unfavorable conditions), the outflow is described by the so-called Pressure Outflow Relationship (POR). The demand is split into three levels of satisfaction: adequate or full, partial or degraded, and critical or zero outflow. Adequate or full outflow appears when the available pressure head is enough to fully satisfy the water requirements for all the consumers. This demand satisfaction level allows to continue operating under DDM. The usefulness of the proposed PDM approach comes in partial or degraded outflow scenarios in which the delivery conditions are given by the current system pressure. In extreme conditions, there is a zero outflow that corresponds to the case in which the system collapses in its operation (partially or in a full manner). In this case, the system does not guarantee delivery of any outflow to the user. POR is able to capture the behavior of the system when it is working under stress conditions. This in terms of showing the pipe flows reordered and so the supply of the outflow available. There are several proposals in the literature related to POR (?; ?; ?; ?; ?; ?; ?). The PDM approach follows Eq. (??).

$$
\mathbf{c}(\mathbf{h})=\mathbf{d} \times\left\{\begin{array}{cl}
1 & \text { if } \mathbf{h}_{\mathbf{s}} \leq \mathbf{h} \\
\left(\frac{\mathbf{h}-\mathbf{h}_{\mathbf{m}}}{\mathbf{h}_{\mathbf{s}}-\mathbf{h}_{\mathbf{m}}}\right)^{0.5} & \text { if } \mathbf{h}_{\mathbf{m}}<\mathbf{h}<\mathbf{h}_{\mathbf{s}} \\
0 & \text { if } \mathbf{h} \leq \mathbf{h}_{\mathbf{m}}
\end{array}\right.
$$

where $\mathbf{h}_{\mathbf{m}}$ corresponds to the minimum head. The value of $\mathbf{h}_{\mathbf{m}}$ is commonly fixed for a system and directly related to the nodal elevation. $\mathbf{h}_{\mathbf{s}}$ refers to the service head necessary to guarantee the consumers' water requirements. Some criterion for $\mathbf{h}_{\mathbf{s}}$ in order to consider the flow directions and the connected pipes' head losses is presented in ?).

The DDM and PDM system can be approached in various ways. For example, by means of a damped Newton method as it is proposed in ?). Another interesting proposal is presented in ?) considering sensitivities with respect to the demand. These local sensitivities provide relevant information on aspects such as sensor placement or confidence intervals for hydraulic performance predictions. Examples of other PDM formulations ?) use a Co-content Model Approach for the hydraulic analysis of WDNs under unfavorable operative conditions. The up-to-date snapshot of 
the ongoing research in this area (software applications for PDM) can be found in (?; ?).

Water pipe burst/leakage. Pipe burst or leakage is a pressure dependent phenomenon and key for WDN resilience assessment. This is a challenging issue as the available pressure at consumer points might decrease with the pressure head reduction due to water leaking at the pipe burst point (?). Pipe burst events cause inefficient energy distribution through the network (?; ?). In addition, low pressure conditions within the system may lead to the introduction of pollutants into WDNs and, consequently, worsen water quality (?). Therefore, a pipe break (via pipe burst) represents not only decreasing revenues for water utilities (?), but also a deterioration in the water quality and wasted energy resources. It should be properly distinguished between pipe burst and pipe leakage. The first is a visible damage and it can start to be fixed immediately (if operational resources are available from the water utility). Leakages usually are not visible and their detection requires mathematical algorithms and sophisticated methods and/or equipment (?).

The treatment of the outflow as pressure head function for a specific junction node is in general based on some form of the orifice equation (?). Thus, in WDNs both leaks and burst are commonly modeled by the well-known Torricelli equation (?). Torricelli's equation represents, under conditions of zero energy loss, the conversion of potential pressure energy to kinetic energy. A discharge coefficient $\left(\mathbf{C}_{\mathbf{d}}\right)$ is included in the Eq. (??) to consider energy losses by friction and the orifice's effective area (?).

$$
\mathbf{c}_{\mathbf{L}}(\mathbf{h})=\left\{\begin{array}{cc}
\mathbf{C}_{\mathbf{d}} \mathbf{A}_{\mathbf{L}} \sqrt{2 g \Delta \mathbf{h}}=\mathbf{C}_{\mathbf{d}} \mathbf{A}_{\mathbf{L}} \sqrt{2 g} \sqrt{\mathbf{h}-\mathbf{h}_{\mathbf{m}}} & \text { if } \mathbf{h}_{\mathbf{m}} \leq \mathbf{h} \\
0 & \text { otherwise }
\end{array}\right.
$$

where $\mathbf{c}_{\mathbf{L}}$ represents the water leaked; $\mathbf{C}_{\mathbf{d}} \mathbf{A}_{\mathbf{L}}$ is the leak effective area; $\mathbf{A}_{\mathbf{L}}$ represents the orifice area; and $g$ is the gravity acceleration. For modeling purposes, the orifice equation is typically simplified as an emitter form where multiple orifices might be combined into a single emitter (?).

\section{AN APPROACH TO RESILIENCE CRITICALITY INDICATORS}

In WDNs, there is an essential need to generate specific metrics that allow to quantify the system performance when it is working under failure (or unfavorable) conditions. There are several 
indicators aiming to capture this performance through mathematical formulations. We propose that these indicators may be classified in the following six groups: 1) Power/Energy (?), 2) Performance (such as the demand satisfaction indicators proposed herein), 3) Graph theory/Social Networks (?), 4) time (?), 5) sensitivities (?), and 6) others ?.

A widely used method to quantify the WDN resilience is through power/energy-based indicators. This indicators class is, at the same time, divided into three groups: a) power-based indicators, b) energy based indicators, and c) entropy-based indicators. The most popular power/energy-based indicator is Todini's resilience index (?). This index is a power-based indicator that describes the relationship between the power supplied to the end-user and the maximum power dissipated in the network to satisfy the water requirements for the consumers. Several authors have proposed other definitions based on the Todini's resilience index (?; ?; ?; ?; ?). These authors attempt to obtain a better understanding of the network reliability through the uses of their indicators. ?) and ?) included into the Todini's indicator the uniformity of pipe diameters and loop diameter, respectively. ?) found an inconsistency in Todini's resilience indicator if it is used to measure resilience when there are multiple sources for the WDN. Subsequently, they propose an indicator attempting to fix this issue. Other modifications of Todini's resilience index aimed to enhance it by including different pressure-dependent modeling cases. This is the case of the proposals of ?) which focuses on leakage related issues and ?) which deals with leakage and consumption issues.

The Franco-German ResiWater Project (?) proposes the use of an event-driven approach that can be classified as a performance indicator. In this project ?) exemplify the network resilience due the occurrence of some critical event and consider aspects of the resilience such as: sequence of events, type of approach used in the hydraulic model (DDM or PDM), the system performance state, and the use of resilience power-based indicators.

The energy-based indicators address resilience assessment through the energy available at the system. Some examples of these indicators are minimum and maximum head sources; the minimum, mean, and maximum node pressure (?); the standard deviation of the node pressures (?); the minimum and the sum of surplus head (?); and the energy dissipation (?), among others. 
The entropy-based indicators propose an interesting point of view with respect to the network resilience assessment. This is the case of the statistical flow entropy indicator proposed by ?) based on the relative uniformity of the pipe flow rates (?). In ?) RCIs of the group of power-based and entropy-based indicators are evaluated for WDN design. The authors evaluated the results of the optimization under critical operating conditions: single isolation action and hydrant service. The paper concludes that the group of power-based indicators represent a better estimation of resilience. ?) proposed other definition of the resilience indicators, where there are considered aspects such as time, water quality (before and after an $\mathrm{AE}$ ), reserve capacity in tanks, and demand satisfaction.

Graph-theory based indicators represent an alternative for WDN resilience assessment (?; ?). ?) and ?) proposed hybrid, hydraulic and graph-theory based, approaches for assessing the WDN resilience. This was done by considering the pipeline's geodesic distances and head losses associated with the water flow. In both papers, the criticality of pipes is measured through the effective supply of users. These approaches were complemented by ?) by assessing WDNs resilience through a topological perspective. There were also proposed to extend the graph-theory based indicators into a multi-scale order in order to take into account the district metered areas (sectors) configuration. ?) show that geometrical and topological features (using graph-theoretic and fractal tools) provide useful knowledge for the WDN resilience assessment even in the case of not having or partial hydraulic information. Also based on a topological metrics, ?) present a graph decomposition model for WDNs. The developed model is used to facilitate WDN reliability analysis (?). Another interesting example, mainly applied on power supply systems, is presented in ?), where the authors proposed using graph theoretical measures for critical link analysis. This is done by a computing reliability of links and an object-oriented based method for vulnerability analysis. It is worth mentioning that the recognition of topological interdependence among critical infrastructure systems (e.g. energy, water supply and wastewater, communications, transport systems) may avoid serious consequences (?) due to the network working under the effects of anomalous events (?).

Impact of a failure - Demand satisfaction RCI. In a system failure scenario it is expected to get a reduction on the volume of water supplied (?). Thus, a proper evaluation of the network 
performance and determining how critical is the reduction in the supply becomes a challenge for water utilities. For a specific time, the satisfaction rate SR in a WDN (Eq. (??)) is the relationship between the water provided and the user's requirements (?; ?). In addition of being an RCI, SR is also an estimation of the reliability when it is computed for a given failure in $j$-th component (or set of components) with respect to its impact at $i$-th user (node).

$$
S R_{i, j}=\frac{c_{i, j}}{d_{i}}
$$

The impact (due to the network operating under unfavorable or critical conditions) is given by the average satisfaction rate (ASR) for the entire system. ASR describes the relationship between the total volume of water supply and the total volume of users demand, Eq. (??). Therefore, ASR is the response of the system in terms of the availability of water when it is operating under unfavorable conditions (due to the failure of the component $j$ ). In Eq. ??, $n_{d}$ is the total number of demand nodes. ASR is ranging between 0 and 1; where 0 is the collapse situation and 1 is the case in which the whole system is totally resilient to the failure.

$$
A S R_{j}=\sum_{i=1}^{n_{d}} \frac{d_{i}}{\sum_{i=1}^{n_{d}} d_{i}} S R_{i, j}=\frac{\sum_{i=1}^{n_{d}} c_{i}}{\sum_{i=1}^{n_{d}} d_{i}} .
$$

In general, overviewing risk assessment modeling for pipes uses break frequency and degradation rates as main aspects for assessing pipe deterioration. The analysis of the state of the entire network pipes requires a detailed information of their characteristics. However, this type of information is not often available in water utilities (?) and the risk quantification analysis is not contemplated herein.

\section{CASE-STUDIES}

This section implements further applications of the methodology for assessing WDN criticality proposed in Section ??. Two WDNs working under abnormal/degraded operating conditions, as 
described above (Section ??), are used for their critical analysis. Firstly, a small size benchmark network is investigated. The second case-study corresponds to a medium size utility network with an average population density in France. The Hazen-Williams (H-W) and the Darcy-Weisbach formulation are used to compute the head loss for the simple and complex case-study, respectively.

\section{Simple Case-Study}

The first case-study corresponds to a two loop network (TLN) proposed by ?). This is a simple benchmark network composed by 6 demand nodes, 8 pipes and 1 reservoir (Fig. ??). The choice of such a simple network is motivated by the necessity of facilitating the analysis of the results. Several authors have used this network for WDN management research and resilience assessment (?; ?; ?; ?). Table ?? details the TLN network features.

\section{Simple Case-Study - Impact at System and Nodes as Consequence of Burst Position}

This case-study investigates three different locations along one affected pipe and how are their effects in the system performance. The results are presented in Fig. ?? and the hydraulic simulations are computed by PDM. Fig. ??a presents the resilience assessment for the ASR indicator. The maximum, medium and minimum impact on the network are estimated by $\max \operatorname{Imp} p_{j}=\min \left(A S R_{j, P o s}\right)$, $m e d \operatorname{Imp}_{j}=\operatorname{mean}\left(A S R_{j, P o s}\right)$ and $\operatorname{minImp}_{j}=\max \left(A S R_{j, P o s}\right)$. The network performance at the system nodes for the three mentioned positions of the burst, in terms of pressure head and water availability (SR), are presented in Fig. ??(b-d) and Fig. ??(e-g), respectively.

Fig. ??a shows that the maximum impact on the network under study occurs when the burst is located at one of the ends of an affected pipe (Fig. ??a; curve maxImp). Pipe P3 presents an exception as the minimum network impact is given by Pos50. At this point it is important to make the proper selection of the leak point for each pipe. This is because the most critical behavior of the network under this failure may significantly vary depending on the burst position along the pipe. Estimating it wrongly can lead to do not take proper actions and consequently to bring cost overruns for water utilities. Fig. ??a also shows a difference for the failure impact at each pipe $\left(\min I m p_{j}-\max \operatorname{Imp} p_{j}\right)$. For pipe P1, the maximum difference in the system performance deterioration is given for the pairwise (Pos0, Pos100). This difference is approximately $91 \%$ 
in terms of water availability resilience. If a burst occurs in the pipe P1, at position Pos0, the resilience for entire system is $97 \%$. We can also observe that the leak outflows for pipe P1 at Pos0 is 2583.8 L/s, at Pos50 is $906.3 \mathrm{~L} / \mathrm{s}$, and at Pos 100 is $650.7 \mathrm{~L} / \mathrm{s}$. The water head loss coming from T1 to the leak position in P1 is $0.1 \mathrm{~m}, 52.6 \mathrm{~m}$ and $56.2 \mathrm{~m}$, respectively. It is worth mentioning that the leakage outflow rate at Pos0 is very high $(2583.8 \mathrm{~L} / \mathrm{s})$, comparing it to Pos50 and Pos 100 . However, the available pressure head downstream the break point is the same than the associated with the reservoir (following the model). Likewise, and due to the head loss for this case, the pressure head available downstream of break point is similar to that obtained for the original state of the network (without failure). This results on a minimal decrease in the system resilience comparing it to the other two position tested. Thus, in future works it is worthwhile to study other indicators of resilience in which the volume of leaked water is considered in the approach.

The maximum impact in the network due to an $\mathrm{AE}$ points out at the most critical allocation of the burst along the affected pipes (Fig. ??a; curve maxImp) will be used in Section ??. This is of main importance for assessing the network performance after the implementation of the proposed PAs (MIA and SIA).

Fig. ??(b-d) shows a considerable decreasing in the state of the system performance as consequence of the pipe burst. Even though some nodes are still working, we can say that the network's performance state is, in general terms, under failure mode. This is strongly confirmed when the pipe burst is placed at one end of the pipe (Pos0 or Pos100). In the absorptive phase of the system resilience, the network performance state is considered in failure mode when the first consumer is affected $\left(\mathbf{h} \leq \mathbf{h}_{\mathbf{s}}\right.$; in our case $\mathbf{h}_{\mathbf{s}}-$ elevation $=30 \mathrm{mH} 2 \mathrm{O}$, green curve $)$

The node performance state is determined by two conditions; $\mathbf{h}_{\mathbf{m}}<\mathbf{h}<\mathbf{h}_{\mathbf{s}}$ (with $\mathbf{h}_{\mathbf{m}}-$ elevation $=0 \mathrm{mH} 2 \mathrm{O}$ in our case, red curve) and $\mathbf{h} \leq \mathbf{h}_{\mathbf{m}}$; for the network working under degraded and failure mode, respectively. Fig. ??(b-d) shows negative pressure values. An incorrect prediction can occur in hydraulic simulations with PDM approach. This is since the mathematical model only restricts delivered water if insufficient pressure values are obtained. Despite this approach will raise the pressure, the PDM model still can yield negative pressure with zero water outflow (Fig. ??e-f) 
as mentioned in ?).

Simple Case-Study - Pipe Burst and Isolation of the Affected Pipe

Fig. ??(a-b) shows the system resilience results for the three operative conditions considered in this paper. The respective rankings of the hydraulic importance of the affected component (in this case pipes) for the simple case-study are shown as well in this figure. Fig. ??(a-b) includes the most critical AE (due to the burst location) for each affected pipe (see Fig. ??a), SIA, and MIA.

The set of adjacent pipes for MIA is obtained through Eq. (??). It should be necessary to consider the implementation of subsequent actions by the water utilities (to be applied after PAs) such as auxiliary pumping (?) at the adaptive resilience stage or the implementation of long-term actions such as repair or to repair/replace (long term actions) at the adaptive or the restorative resilience stage, respectively.

The values of the resilience previously obtained (Fig. ??a, maxImp curve) for the average impact at system are ordered from major to minor impact on the resilience assessment. This ordered curve is presented in Fig. ??(a-b; blue line). The performance results for the network working under effects of the isolation PAs (SIA and MIA) are showed in Fig. ??(a-b); Fig. ??a with red continuous line for SIA, and Fig. ??b with red dashed line for MIA. For SIA and MIA results, the order obtained with the ranking of pipes for the network working under effects of the $\mathrm{AE}$ is preserved. Fig. ??(c-e) shows the average impact at the system nodes generated for the network working under effects of the three adverse operative conditions evaluated.

The comparison between the AE with each tested isolation PA (see Fig. ??) shows that:

- in both of the PAs evaluated (SIA and MIA), we can observe that the internal vulnerability of pipes (1 - ASR) is higher for AE than PAs at $t_{\text {pall }}$ (Fig. ??,a-b). This is valid for the components that do not collapse the network by themselves.

- SIA shows better results (as isolation action) than MIA as it provides the maximum enhancement, $71 \%$, for the network resilience. This is an outstanding result as the operative condition when MIA shows a maximum enhancement for the network resilience of just 
26\%. However, as mentioned in Section ??, SIA for each pipe is not a realistic solution (due to the potential cases of pollutants into the network, to the valves location, that is non-profitable in economic terms, among others). In this sense, the set of pipes associated with the pipes P4 to P8 are shown as an option that would not collapse the network, it just in case that is necessary to isolate an specific area of the network. In the case of SIA, only the isolation of the pipe P1 is capable to collapse the system's functioning.

- Fig. ??a shows a system resilience enhancement after the implementation of SIA. The resilience increases in average up to $55 \%$ for all the pipes except for P1, the pipe associated with the tank.

- Fig. ??(c-e) shows that the most critical impact on the simple case-study nodes (due to the AE and PAs) occurs in the farthest nodes (N6 and N7) from the tank. Nonetheless, we can observe that although the farthest node (N7) from the tank is not the node that suffers the most impact in the three operative conditions evaluated and the most critical node is node N6 (see Fig. ??d). This reveals that selecting a suitable level of detail (i.e. node elevation, water demand, pipe length and diameter) for hydraulic models is a relevant issue to be considered on the network resilience assessment.

\section{Complex Case-Study}

In this section we apply the proposed methodology for criticality assessment of WDNs through a utility network case-study. This WDN (Fig. ??) has 1738 nodes, 1 tank, and 1781 pipes (116 Km), 43 loops, and 3 different pipe materials (cast-iron, PVC, PE). The total demand ranges from $5.7 \mathrm{~L} / \mathrm{s}$ to $21.3 \mathrm{~L} / \mathrm{s}$ and the demand curve is defined at hourly basis for a week (Fig. ??b). The head of the tank is considered as a reservoir for the related hydraulic simulations and its upstream network is not taken into account further.

\section{Complex Case-Study - Impact at System and as Consequence of Burst Position}

A map of component importance represents the average system impact as consequence of the $\mathrm{AE}$ in each pipe of the system. The resilience results presented as component importance maps 
with respect to the maximum and minimum demand (see Fig. ??b) are approached in Fig. ?? by showing the ASR indicator. The AE is tested at three different positions along each pipe. Thus, three different maps are built (for each demand evaluated) depending on the AE's average impact in the system. The AE is located at the middle of each pipe for better comparison between maps.

Fig. ?? encompasses four analysis areas aiding further results interpretation. As consequence of the AE, Fig. ??(d-f) shows a notable decreasing on the network resilience for zone1, in comparison with the estimated resilience for the maximum demand period (Fig. ??,a-c) for the same zone. This effect is due to a pressure head increment as the demand nodes requirements decrease (minimum peak of demand). However, this performance is not preserved in the maxImp maps (insets c and f) for zone2. Fig. ?? also shows two critical zones (zone2 and zone3) for all the evaluated characteristics (demand peaks and burst position). The area nearby the reservoir (zone4) shows a not considerable affection with respect to the entire system resilience for the AE, also for all the evaluated conditions. This performance is due to the redundant topology of pipes belonging to zone4 (Fig. ??c). Only a small area in zone4 is sensible to the AE. Still, there is a small effect generated in such zone by the $\mathrm{AE}$ in terms of the entire network performance.

Table ?? shows a maximum impact in resilience for the considered AE in the period of minimum demand. The network performance is, then, consequence of an increasing pressure head due to decreasing demand requirements. A greater impact for the AE of burst is shown in the periods of low demand making them the most critical periods for this event. Overall, the results show a variation between the minimum (44.68\%) and maximum (99.39\%) resilience for all the network characteristics contemplated (maximum and minimum demand peak and position of burst in the pipe). The most critical condition for the network occurs for a value order of $44.68 \%$ (Table ??) in terms of the satisfied average demand.

\section{Complex Case-Study - Pipe Burst and Isolation of the Affected Pipe}

The component importance maps are shown in Fig. ?? for the two isolation PAs considered herein. The zones proposed in Fig. ??, for the AE, are preserved in Fig. ?? to facilitate the comparison between AE and PAs. 
In Fig. ?? we can observe lower dispersion and lower intensity in the average impact suffered by the network after the AE due to the application of the proposed PAs. This is in comparison with the component importance maps obtained for the AE of burst (Fig. ??). A low dispersion means that fewer pipes are capable of generating a considerable impact in the system. Thus, the hydraulic importance for pipes is concentrated at specific points of the network. The intensity in the maps is directly related to the impact suffered in the system due to the closure of the associated area components. In this paper low intensity is interpreted in terms of low resilience. The results are detailed as follows:

- for zone1 both PAs (minimum valley and maximum peak of demand evaluated), present a smaller dispersion than that obtained for the AE at maxImp (Fig. ??; inset c for maximum peak, and inset $\mathrm{f}$ for minimum valley). The intensity is shown to be larger for PA than for the AE. This occurs in all cases except for the pairwise of dashed red circles (Fig. ??), whose intensity is larger for AE than PAs. We compare zone1 at the maximum and minimum demand requirements for each PA. The results show that the dispersion is very similar in all cases but the intensity is often greater for the periods of minimum demand.

- Zone2 and zone3 are considered as the most critical areas for an AE. The results show that zone 2 preserves zone critical characteristics, whilst zone3 becomes into a not significantly affected area for both PAs. This means that the impact of an AE is effectively mitigated with the application of any of the two PAs proposed. Thus, the resilience for zone 3 can be improved through the implementation of actions providing a fast AE detection and isolation of the affected components. At zone 2 the dispersion is preserved for PAs. Thus, not new components acquire hydraulic relevance after the implementation of PAs. Although the intensity has been reduced for this zone with the application of PAs (SIA better than MIA) the results show that PAs of isolation are not considerably effective to mitigate the impact related to AE (specially for the period of maximum demand). There are required adaptive actions and the consideration of "twining" pipes (creating loops for increasing the topological redundancy of the network) for improving resilience. 
- Neither AE of burst nor the application of PAs have a significant impact on the system's operation for zone4.

\section{CONCLUSIONS}

This paper contributes to progress on the implementation and quantification of the conceptual development of water distribution networks (WDNs) resilience. This is done by investigating the consequences of failure in the network components. The proposal is based on the three system capacities and focuses on the absorptive resilience phase of a pipe burst (anomalous event, AE). In addition, it takes into account two palliative actions (PAs) to mitigate any negative AE's effects. The first action is based on the exclusive isolation of the affected pipe (single isolation action - SIA). The second action considers the isolation of the surrounding area of the affected pipe (multiple isolation action - MIA). The impact on the network performance is evaluated for the two cases through the water demand satisfaction.

The paper introduces a novel tool-set for resilience indicators development. This counts on both network structure and energy's level availability. This is possible thanks to the hydraulic model used (Pressure driven model - PDM). The tool-set is tested by assessing the resilience of two case-studies. The proposed methodology is able to suitably identify the most critical components of a network working under unfavorable conditions (AE, SIA, and MIA). In addition, the information is presented by a novel visualization method showing component importance maps. This method has the advantage of allowing better results interpretation than only using numeric information. Component importance maps do not require user experience and can be used both to plan adaptive actions and to redesign the network.

The two case-studies highlight how important is to consider the burst position in the pipe both for practical network management and theoretical developments. Furthermore, estimating the burst position wrongly can lead to do not take proper actions and consequently bring cost overruns for water utilities. The results show the viability of using the analysis of the event in the absorptive phase of the system resilience as an assessment tool both for simple and complex cases. In addition, they lead to the following conclusions and recommendations: 
- The isolation PAs for an AE point out SIA as the ideal isolation in comparison to MIA for palliative actions. The results can be used for recommendations for valve placement. It is recommended a configuration of isolation valves avoiding further network collapse. In addition, if the closed zones are too large the system performance during isolation might be highly affected.

- The results show that the effect of an anomalous event depends on the water demand. The most critical condition of the network performance occurs in conditions of minimum water demand. However, the PAs show a more critical behavior at the period of maximum water demand. Therefore, given that the duration of the isolation of the affected area may be longer (depending on the characteristics of the affected pipe) than the time of the leak, it is recommended that adaptive actions should be evaluated for the maximum peak of demand.

- The comparison of the AE and the first action carried out for the water utility in the specific time evaluated (time in which the first action is implemented in the system), has allowed to generate possible system responses. The overall recommendation is to focus, firstly, on those components that really require adaptive actions or some specific redesign, given the inability of the network to compensate the losses of resilience due to the event.

The paper opens a research avenue for analyzing further the synergy between resilience key performance indicators at different resilience stages. Additional investigation will be necessary for assessing resilience of large-size networks divided in district metered areas. 


\section{APPENDIX I. DETECTION OF SURROUNDING AREA}

This Subsection proposes labeling the sets of three proposed operative conditions of failure: AE, SIA, and MIA. The label directly marks the affected pipe among the set of links $E\{E 1, \ldots, E m\}$. MIA involves the isolation of an entire network area. The surrounding area indicator (termed by the author), sai, label the isolation pipes associated with MIA, Eq. (??).

$$
s a i_{j}=\left|\left(A_{(*, j)}^{N}\right)^{\mathbf{T}}\right|\left|\mathbf{A}^{\mathbf{N}}\right|,
$$

where $j$ represents the link under operative conditions of failure; $(\cdot)^{\mathbf{T}}$ denotes matrix transposition; and $|\cdot|$ represents the matrix of the absolute value of its elements, and $A_{(*, j)}$ is the column extracted from $\mathbf{A}$ indexed by $j$.

The row vector sai identifies the pipes that might be isolated in the network. The values obtained through Eq. ?? are 0,1 and 2. A value equal to 2 refers to the affected pipe by an AE; a value equal to 1 identifies the surrounding pipes (to be isolated); and a value equal to 0 stands for pipes that are not involved in the current action (pipes that not require to be isolated). An instance of the surrounding area identification methodology (through the use of Eq. ??) is shown in Fig. ??.

It is possible to obtain the set of pipes to be isolated as consequence of the failure of $j$ for MIA. This is by extracting the column number of the values different to zero from sai and, subsequently comparing these values with the list of pipes. For Fig. ??, we have the failures in pipe P4 represented as $E_{4}=\{\mathrm{P} 3, \mathrm{P} 4, \mathrm{P} 5, \mathrm{P} 7, \mathrm{P} 8\} . E_{4}$ is the set of adjacent links to the nodes $\mathrm{N} 4$ and N5, pipe $\mathrm{P} 4$ end nodes. In case of having adequate information regarding valve locations, this methodology might be adapted for identification of real pipes that can ultimately be isolated in the network.

\section{Data Availability}

Some or all data, models, or code generated or used during the study are available from the corresponding author by request. 


\section{Acknowledgments}

The work presented in the paper is part of the French-German collaborative research project ResiWater that is funded by the French National Research Agency (ANR; project: ANR-14-PICS0003) and the German Federal Ministry of Education and Research (BMBF; project: BMBF13N13690). 
TABLE 1. TLN network. Node and pipe properties.

\begin{tabular}{|c|c|c|c|c|c|c|c|c|c|c|c|}
\hline \multicolumn{3}{|c|}{ "Nodes } & \multicolumn{6}{|c|}{ Pipes } & \multicolumn{3}{|c|}{ Burst nodes } \\
\hline \multirow[t]{2}{*}{ ID } & \multirow{2}{*}{$\begin{array}{l}\text { elev. } \\
\text { (m) }\end{array}$} & \multirow{2}{*}{$\begin{array}{l}\text { dem. } \\
(\mathrm{L} / \mathrm{s})\end{array}$} & \multirow[t]{2}{*}{ ID } & \multirow{2}{*}{$\begin{array}{c}\text { initial } \\
\text { node }\end{array}$} & \multirow{2}{*}{$\begin{array}{l}\text { term. } \\
\text { node }\end{array}$} & \multirow{2}{*}{$\begin{array}{l}\text { length } \\
\text { (m) }\end{array}$} & \multirow{2}{*}{$\begin{array}{l}\text { diam. } \\
(\mathrm{mm})\end{array}$} & \multirow{2}{*}{$\begin{array}{l}\text { H-W } \\
\text { coef. }\end{array}$} & \multicolumn{2}{|c|}{ Position } & \multirow[t]{2}{*}{$C_{d}$} \\
\hline & & & & & & & & & Pos0 & Pos 100 & \\
\hline $\mathrm{T} 1$ & 210 & - & P1 & $\mathrm{T} 1$ & $\mathrm{~N} 2$ & 1000 & 400 & 130 & $\mathrm{~T} 1$ & $\mathrm{~N} 2$ & 0.6 \\
\hline $\mathrm{N} 2$ & 150 & 27.8 & P2 & $\mathrm{N} 2$ & N3 & 1000 & 400 & 130 & $\mathrm{~N} 2$ & N3 & 0.6 \\
\hline N3 & 160 & 27.8 & P3 & $\mathrm{N} 2$ & N4 & 1000 & 300 & 130 & N2 & N4 & 0.6 \\
\hline N4 & 155 & 33.3 & P4 & N4 & N5 & 1000 & 400 & 130 & N4 & N5 & 0.6 \\
\hline N5 & 150 & 75.0 & P5 & N4 & N6 & 1000 & 300 & 130 & N4 & N6 & 0.6 \\
\hline N6 & 165 & 91.7 & P6 & N6 & N7 & 1000 & 400 & 130 & N7 & N6 & 0.6 \\
\hline N7 & 160 & 55.6 & P7 & N3 & N5 & 1000 & 400 & 130 & N3 & N5 & 0.6 \\
\hline - & - & - & P8 & N5 & N7 & 1000 & 400 & 130 & N5 & N7 & 0.6 \\
\hline
\end{tabular}


TABLE 2. Resilience results - AE of burst

\begin{tabular}{|c|c|c|c|c|}
\hline \multirow[t]{2}{*}{ Demand } & \multirow{2}{*}{$\begin{array}{l}\text { Fig. ?? } \\
\text { Inset }\end{array}$} & \multicolumn{3}{|c|}{ Impact } \\
\hline & & minimum & medium & maximum \\
\hline \multirow{3}{*}{ maximum } & a. & 99.39 & 96.27 & 51.60 \\
\hline & b. & 99.39 & 96.07 & 51.33 \\
\hline & c. & 99.39 & 95.55 & 51.07 \\
\hline \multirow{3}{*}{ minimum } & d. & 99.27 & 96.12 & 45.28 \\
\hline & e. & 99.27 & 95.93 & 44.98 \\
\hline & f. & 99.27 & 95.43 & 44.68 \\
\hline General & - & 99.39 & 95.90 & 44.68 \\
\hline
\end{tabular}




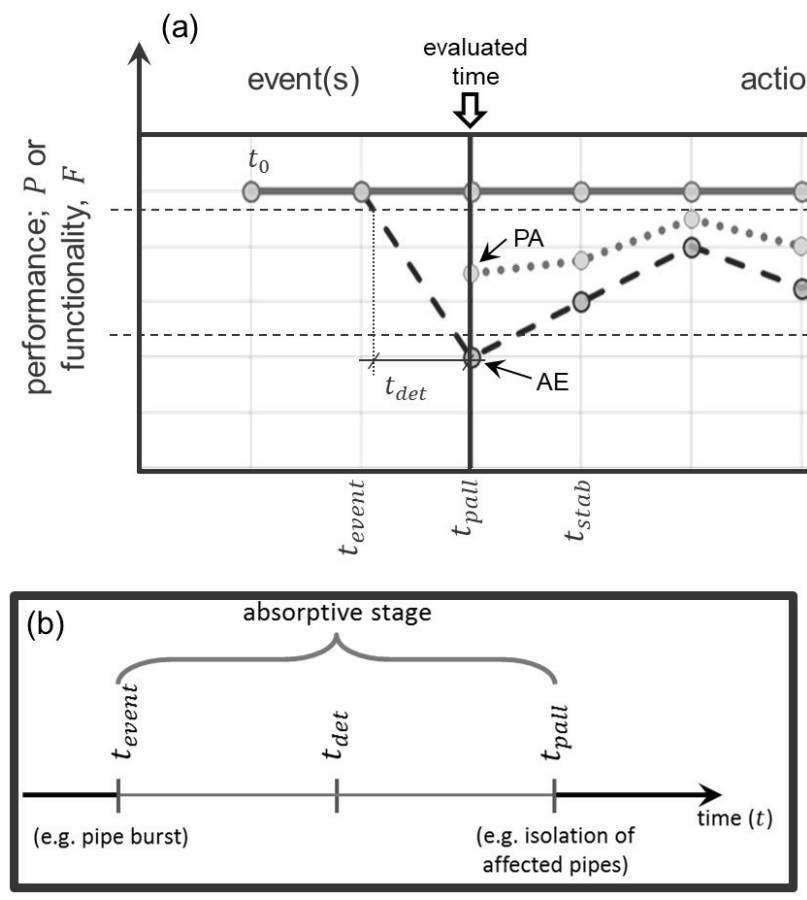

action(s)

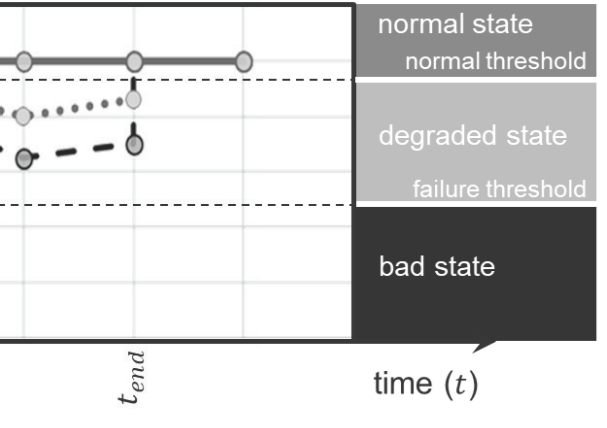

- Event-actions division

Ideal curve (normal operation of the

- - system), or target curve

(with ideal implemented actions)

100 Curve with implemented actions

-O- Anomalous event curve (without implemented actions)

Fig. 1. Anomalous events in a WDN - Resilience curve. (a) Temporal-technical dimension of the resilience, and (b) absorptive phase - times. 
Stage 1. Network and consumer characteristics

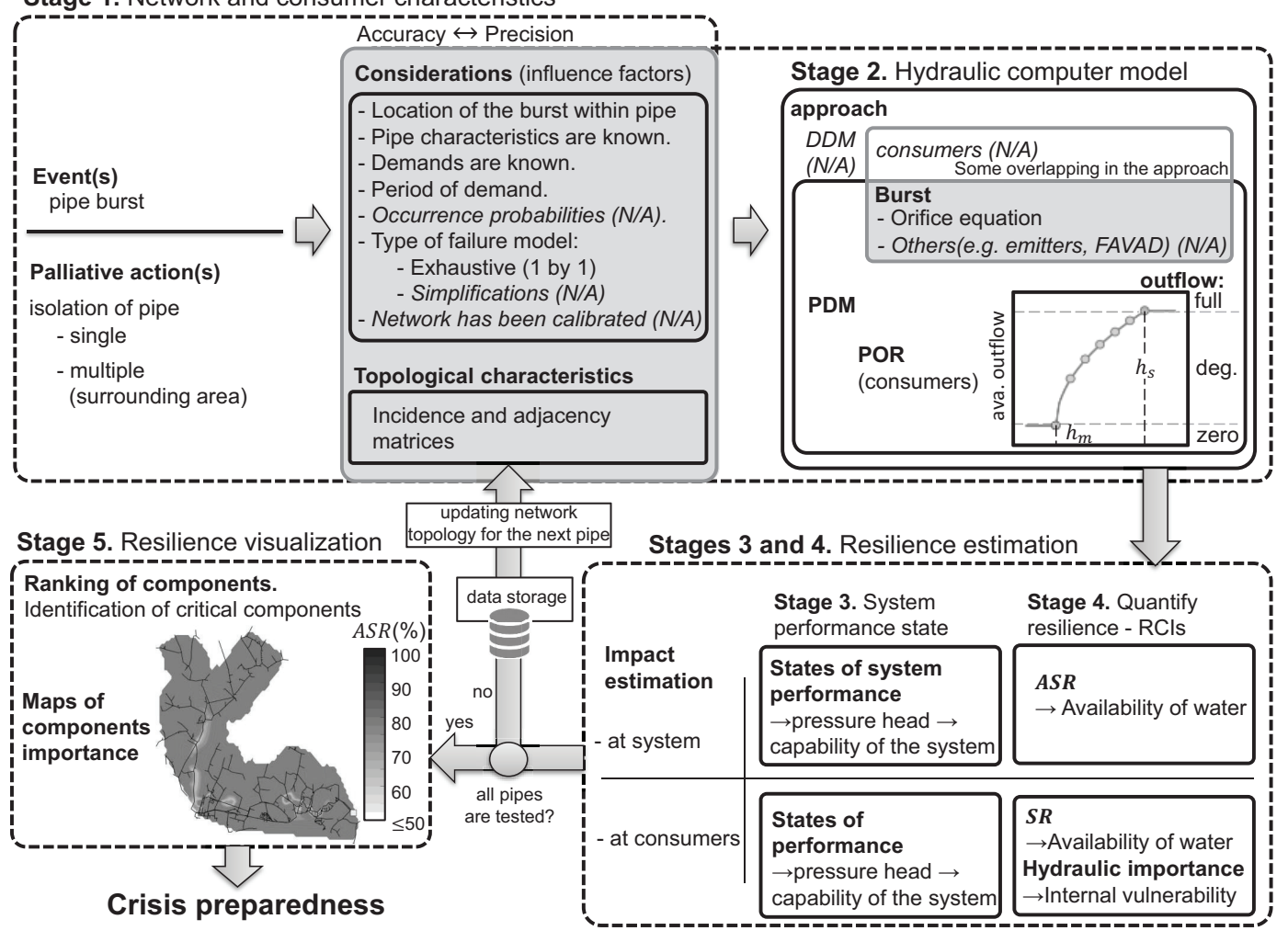

Fig. 2. Overview of the proposed network resilience quantification process. 


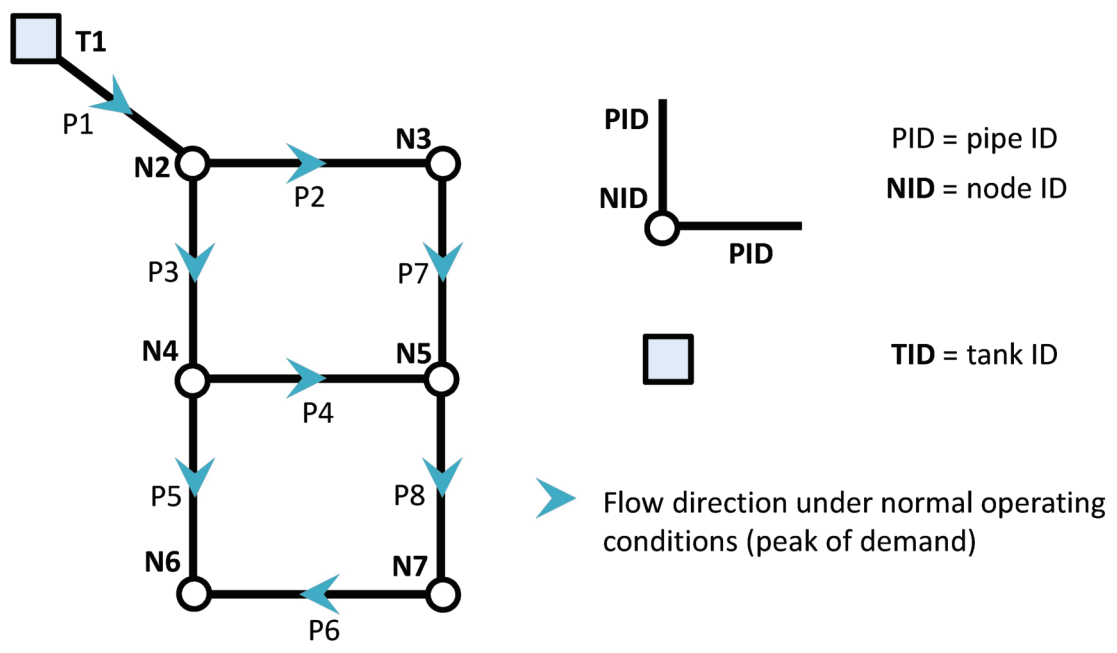

Fig. 3. TLN network. Network layout configuration, and flow directions. 

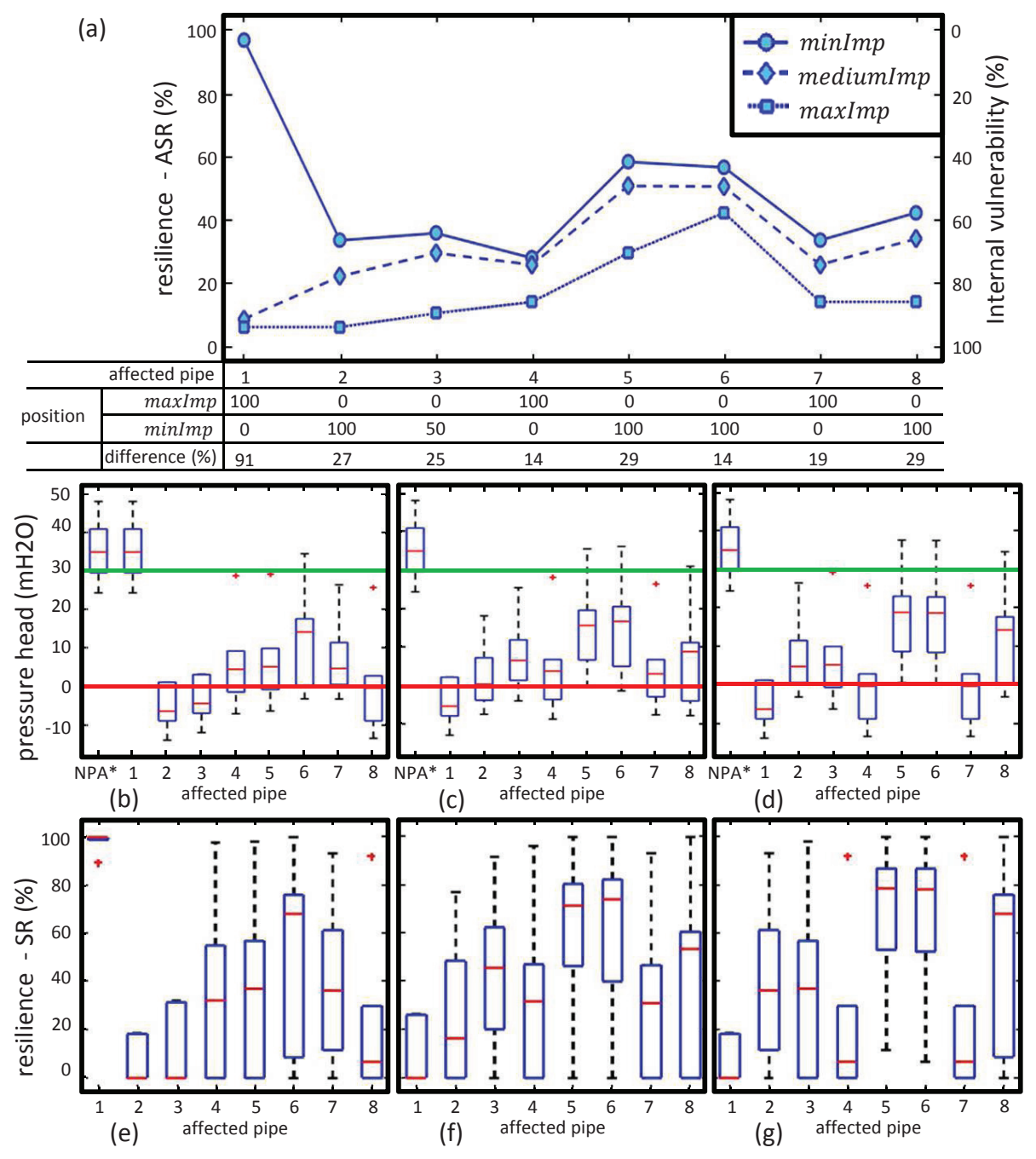

Fig. 4. TLN network - pipe burst. (a) Network impact, (b-d) pressure heads and (e-g) water availability. (b and e) Pos0, (c and f) Pos50, and (d and g) Pos100. NPA*: Non-pipe affected. 

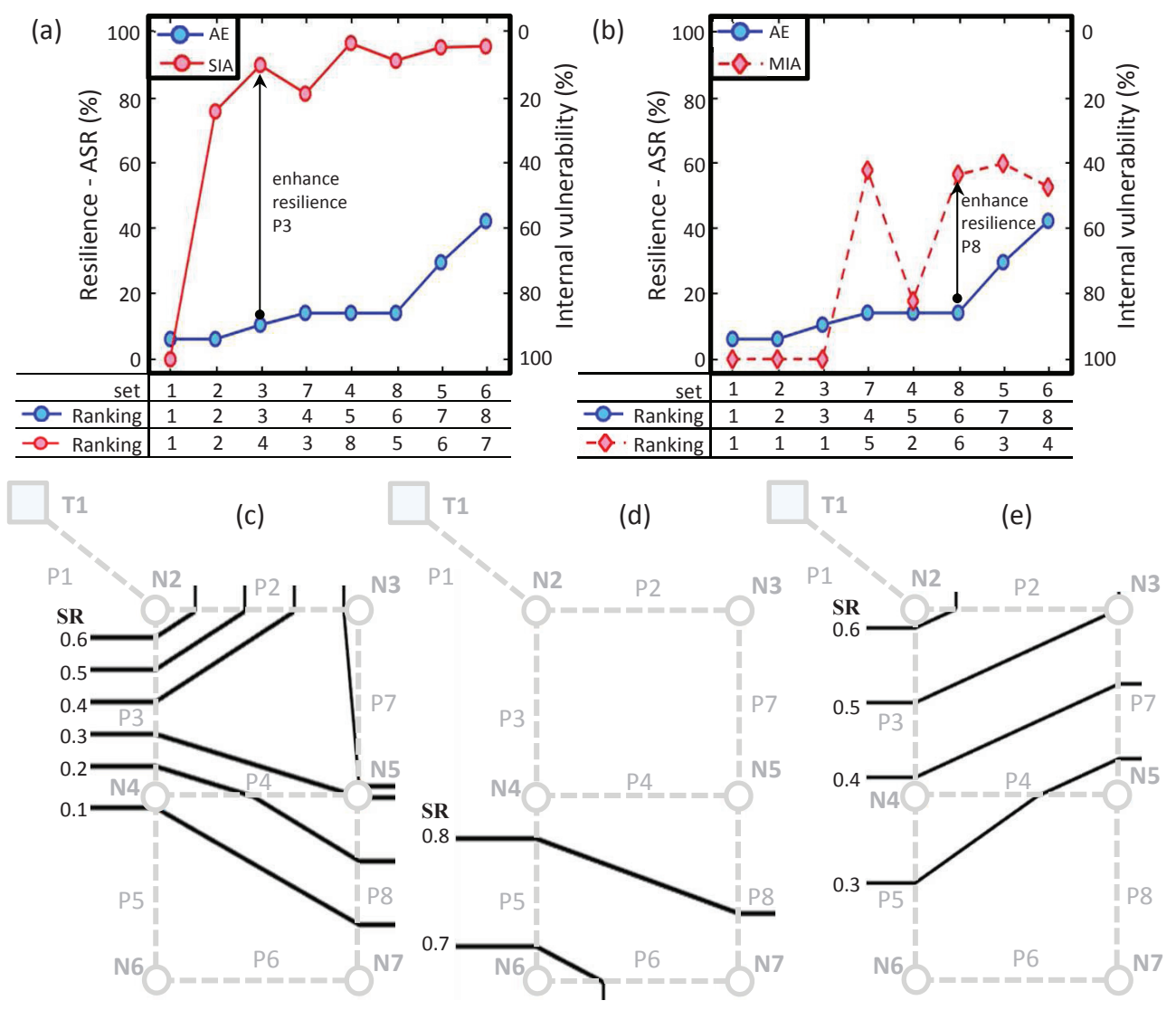

Fig. 5. Burst event and isolation actions - TLN network. (a-b) Impact of the failure, and (c-e) $S R$ isoclines. (a) AE and SIA, (b) AE and MIA, (c) AE, (d) SIA, and (e) MIA. 


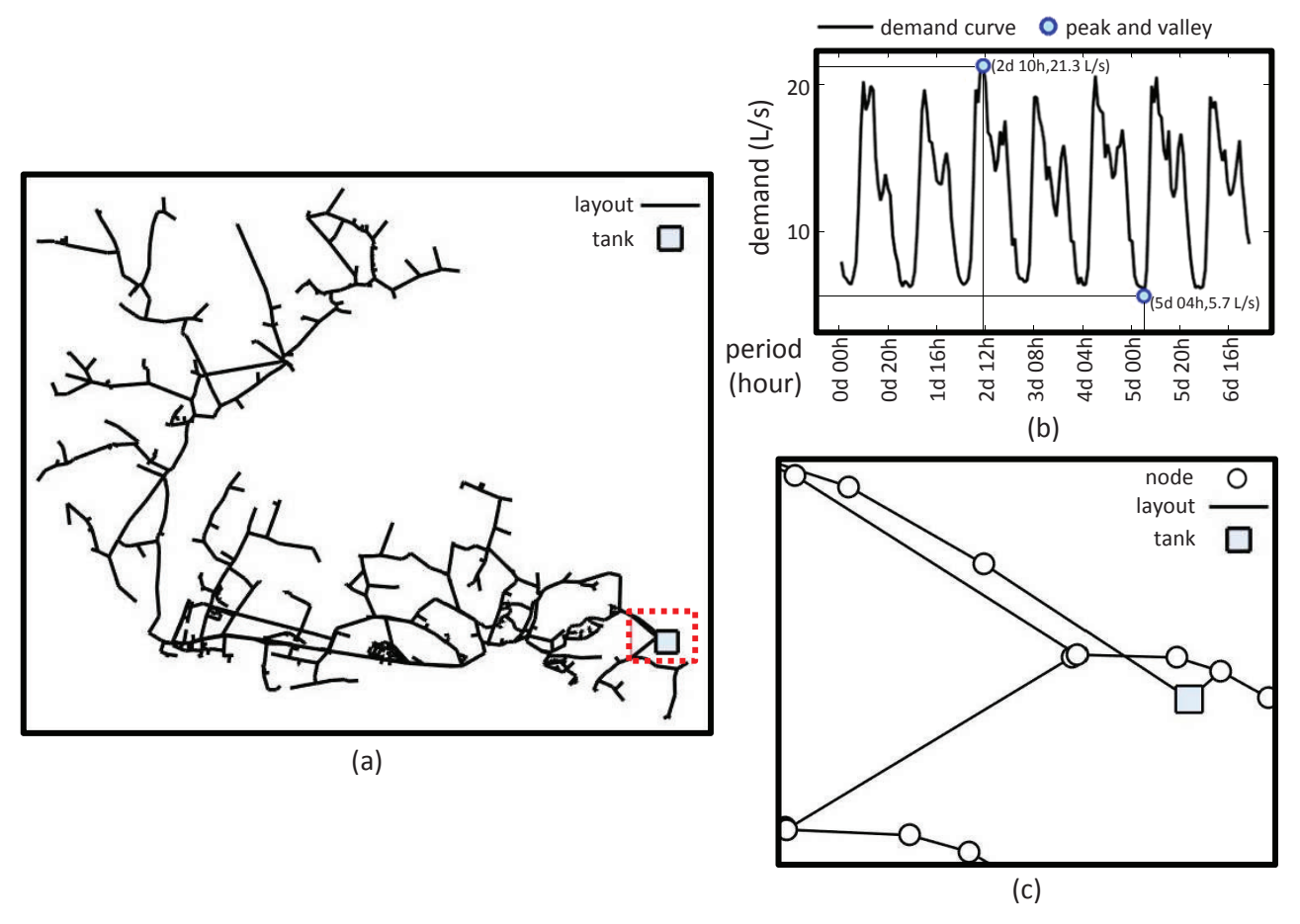

Fig. 6. Complex case-study. (a) Network layout, (b) demand curve, and (c) zoom of red dashed rectangle - nearby area for the tank. 


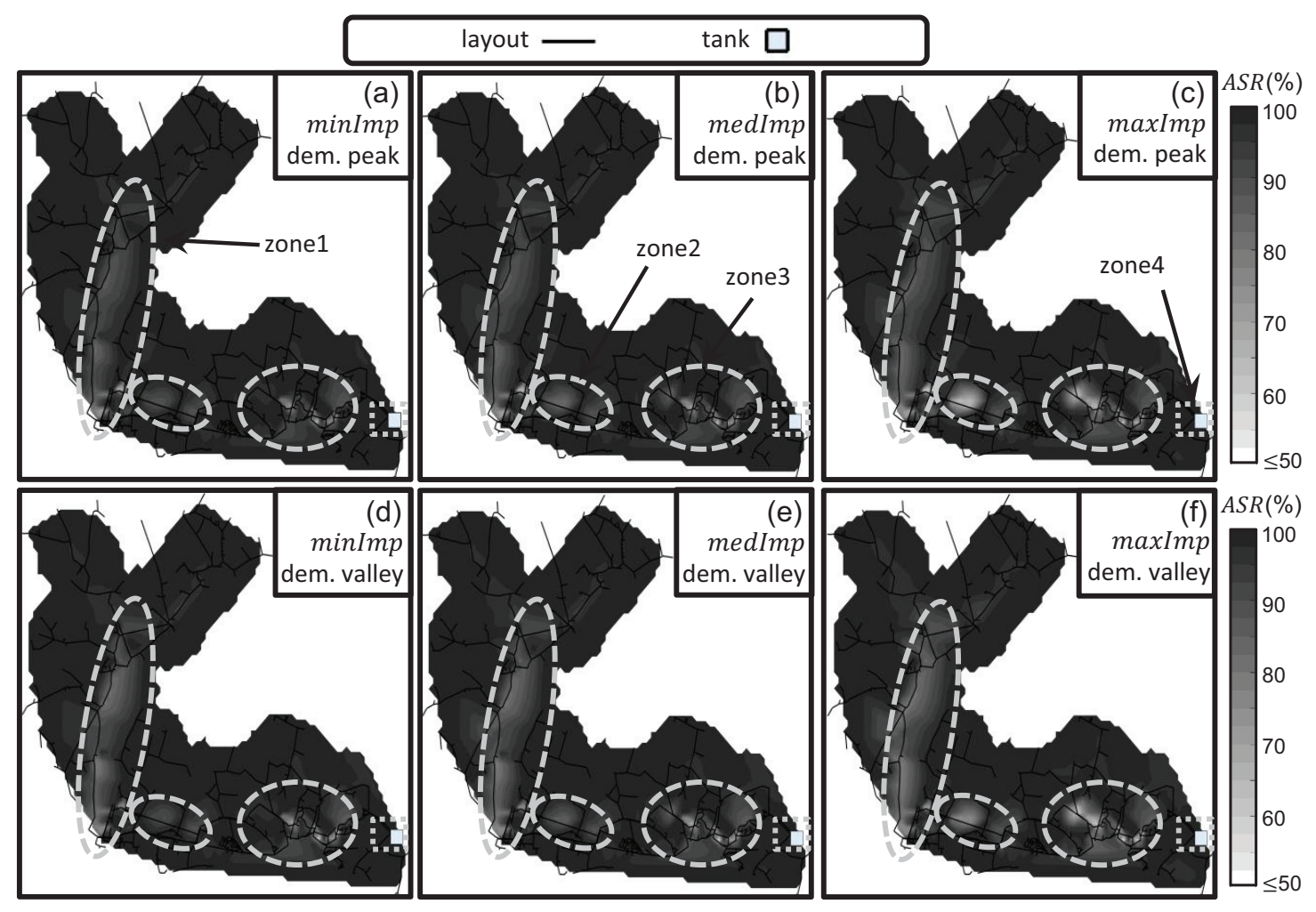

Fig. 7. Resilience based on $A S R$, maps of component importance for $\mathrm{AE}$ of Burst. Quantification of the average impact - complex case. (a-c) Maximum peak of demand, and (d-f) minimum valley of demand. (a and d) minImp, (b and e) medImp, and (c and f) maxImp. 


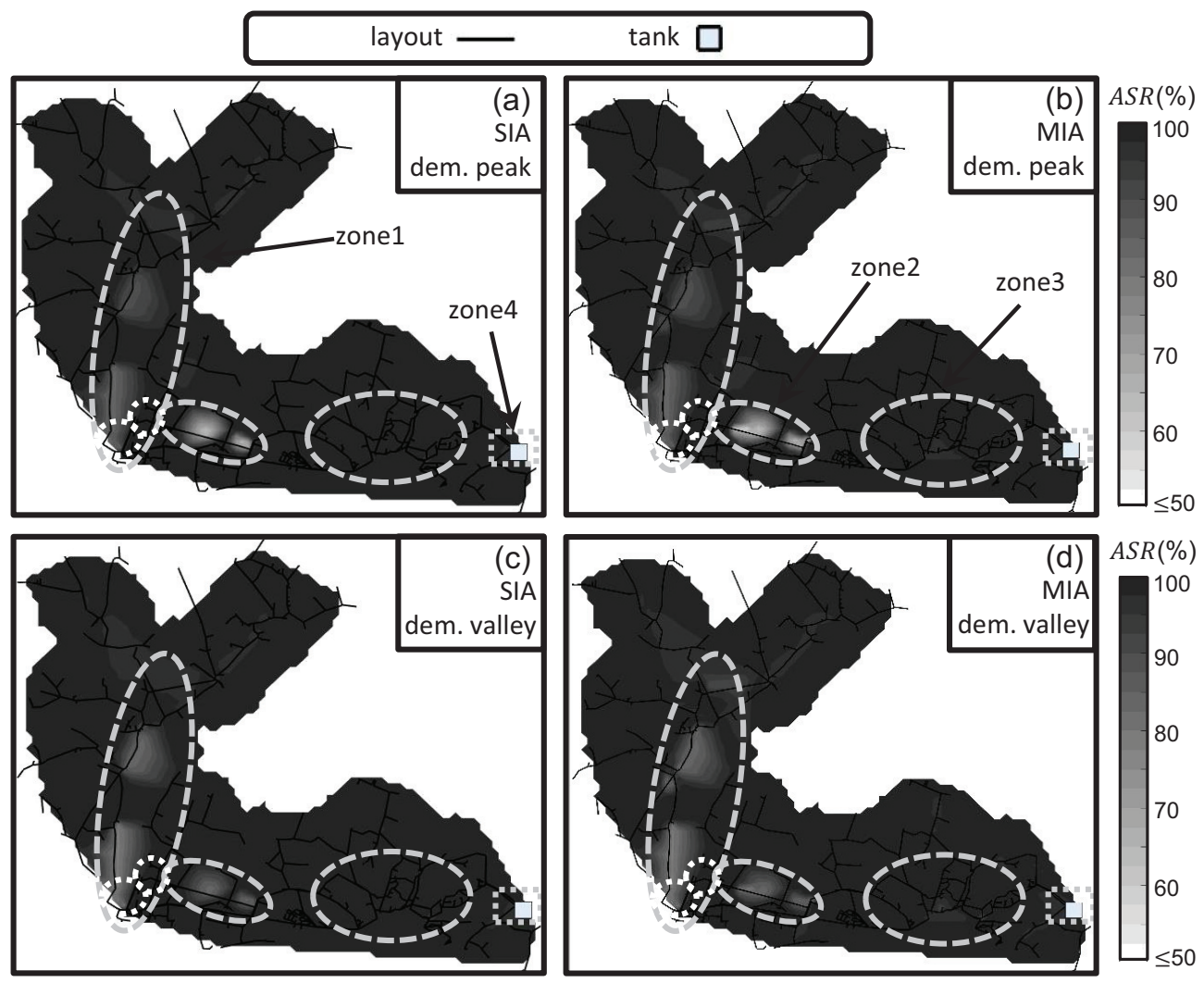

Fig. 8. Resilience based on $A S R$, component importance maps for SIA and MIA. Quantification of average impact - complex case. (a-b) Maximum peak of demand, and (c-d) minimum valley of demand. (a and c) SIA, and (b and d) MIA. 


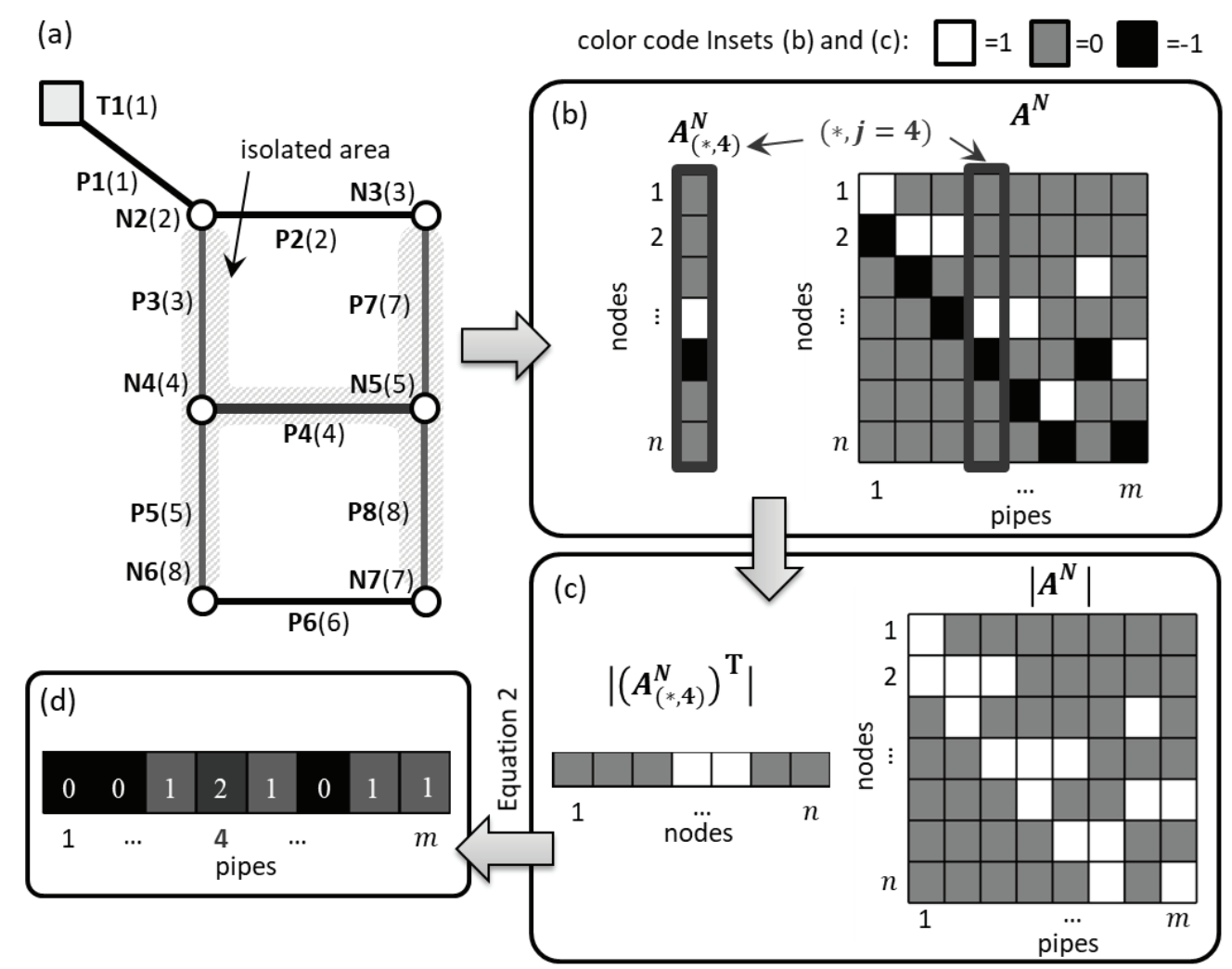

Fig. 9. Surrounding area identification. Example of application of the Eq. ?? applied to a particular pipe (P4 in this case). (a) Network layout, (b) incidence matrix and its respective column vector for the affected pipe, (c) undirected incidence matrix and its respective undirected column vector (transposed) for the affected pipe, and (d) surrounding area indicator. 